\title{
The ancient stellar population of M 32: RR Lyrae variable stars confirmed ${ }^{\star}$
}

\author{
G. Fiorentino ${ }^{1}$, R. Contreras Ramos ${ }^{2}$, E. Tolstoy ${ }^{3}$, G. Clementini ${ }^{1}$, and A. Saha ${ }^{4}$
}

\author{
1 INAF-Osservatorio Astronomico di Bologna, via Ranzani 1, 40127 Bologna, Italy \\ e-mail: giuliana.fiorentino@oabo.inaf.it \\ 2 Dipartimento di Astronomia, Universitá degli studi di Bologna, via Ranzani 1, 40127 Bologna, Italy \\ 3 Kapteyn Astronomical Institute, University of Groningen, Groningen, The Netherlands \\ ${ }^{4}$ NOAO, PO Box 26732, Tucson, AZ 85726, USA
}

Received 7 June 2011 / Accepted 15 December 2011

\begin{abstract}
Using archival multi-epoch ACS/WFC images in the $F 606 \mathrm{~W}$ and $F 814 \mathrm{~W}$ filters of a resolved stellar field in Local Group dwarf elliptical galaxy M 32 we have made an accurate colour-magnitude diagram and a careful search for RR Lyr variable stars. We identified 416 bona fide RR Lyr stars over our field of view, and their spatial distribution shows a rising number density towards the centre of M 32. These new observations clearly confirm the tentative result of Fiorentino et al. (2010b, ApJ, 708, 817), on a much smaller field of view, associating an ancient population of RR Lyr variables to M 32. We associate at least 83 RR Lyr stars in our field to M 32. In addition the detection of 4 anomalous Cepheids with masses in the range 1.2-1.9 $M_{\odot}$ indicates the presence of relatively young, 1-4 Gyr old, stars in this field. They are most likely associated to the presence of the blue plume in the colour-magnitude diagram. However these young stars are unlikely to be associated with M 32 because the radial distribution of the blue plume does not follow the M 32 density profile, and thus they are more likely to belong to the underlying M 31 stellar population. Finally the detection of 3 Population II Cepheids in this field gives an independent measurement of the distance modulus in good agreement with that obtained from the RR Lyr, $\mu_{0}=24.33 \pm 0.21 \mathrm{mag}$.
\end{abstract}

Key words. Local Group - stars: variables: RR Lyrae - galaxies: individual: M 31 - stars: horizontal-branch galaxies: individual: M 32

\section{Introduction}

The compact dwarf elliptical galaxy M 32 is one of the few elliptical galaxies close enough that, with the help of HST, direct observations can be made of its ancient resolved stellar population (e.g., Grillmair et al. 1996; Alonso-García et al. 2004; Fiorentino et al. 2010b; Monachesi et al. 2011). Another example is the peculiar giant elliptical galaxy Cen A, at a distance of $3.8 \mathrm{Mpc}$, where the red giant branch (RGB) and red clump (RC) have been resolved in the outer halo (e.g., Rejkuba et al. 2011). More distant elliptical galaxies, and also the extremely high surface brightness inner regions of M 32, can only be studied in integrated light (e.g., Renzini 2006; Rose 1994; Trager et al. 2000; Coelho et al. 2009). Integrated light provides a picture of the mean properties of a galaxy, but these analyses are rarely unique and cannot avoid a strong bias from the dominant stellar population.

The search for traces of an old (>10 Gyr) stellar population is the main motivation for this study of the resolved stellar population of M 32. RR Lyr variable stars are unequivocal indicators of the presence of a stellar population $>10 \mathrm{Gyr}$ old. There have been a number of detailed studies of the RR Lyr population of M 31, both with ground-based wide field surveys of the halo (Pritchet \& van den Bergh 1987; Dolphin et al. 2004), and HST monitoring of M 31 globular clusters (Clementini et al. 2009)

* Full Table 2 is available in electronic form at the CDS via anonymous ftp to cdsarc.u-strasbg. fr $(130.79 .128 .5)$ or via http://cdsarc.u-strasbg.fr/viz-bin/qcat?J/A+A/539/A138 and small halo and disk fields (Brown et al. 2004; Jeffery et al. 2011). There have also been studies in the region around $M 32$ (Alonso-García et al. 2004; Sarajedini et al. 2009; Fiorentino et al. 2010b).

A recent analysis of the resolved stellar population of M 32 using the ACS/HRC camera, which has a tiny field of view $\sim 30 \times 30$ arcsec square (see small blue square in Fig. 1) but very high spatial resolution (a factor two better than WFC/ACS), concluded that the bulk of the stellar population has an age in the range $8-10$ Gyr old, with a mean metallicity, $[\mathrm{Fe} / \mathrm{H}] \sim-0.2$ dex (Monachesi et al. 2011). However their colour-magnitudediagram (CMD) did not reach the main sequence turn off stars of the oldest stellar population. They also associated a faint blue plume (BP) of stars in the CMD to $\geq 1$ Gyr old stars in M 32 . Using the same ACS/HRS data 17 RR Lyr were found, and it was tentatively suggested that $7_{-3}^{+4}$ could be associated to M 32 (Fiorentino et al. 2010b). The small field of view made this result highly uncertain simply due to the poor spatial sampling of a sparse population and the significant contamination from M 31 . Here we use archival ACS/WFC data, with significantly better spatial coverage (see large red square in Fig. 1), to revisit the detection of RR Lyr associated with M 32.

\section{Data reduction and analysis}

We used 36 archival ACS/WFC images ${ }^{1}$, covering a $3.3 \times$ 3.3 arcmin square region, centred at $004256+405050$ (J2000),

\footnotetext{
${ }^{1}$ Proposal id: 9392; PI: M. Mateo.
} 
Table 1. Log of the M 32 ACS/WFC archival data.

\begin{tabular}{lccccc}
\hline \hline Field & $\alpha_{\mathrm{J} 2000.0}$ & $\delta_{\mathrm{J} 2000.0}$ & Filter & Exposure time & Date \\
\hline F1 & $00^{\mathrm{h}} 42^{\mathrm{m}} 56^{\mathrm{s}}$ & $+40^{\circ} 50^{\prime} 50^{\prime \prime}$ & $F 606 W$ & $8 \times 1000$ & 24 Nov. 2004 \\
F1 & $00^{\mathrm{h}} 42^{\mathrm{m}} 56^{\mathrm{s}}$ & $+40^{\circ} 50^{\prime} 50^{\prime \prime}$ & $F 606 W$ & $5 \times 1000$ & 25 Nov. 2004 \\
F1 & $00^{\mathrm{h}} 42^{\mathrm{m}} 56^{\mathrm{s}}$ & $+40^{\circ} 50^{\prime} 50^{\prime \prime}$ & $F 606 W$ & $5 \times 1000$ & 10 Dec. 2004 \\
F1 & $00^{\mathrm{h}} 42^{\mathrm{m}} 56^{\mathrm{s}}$ & $+40^{\circ} 50^{\prime} 27^{\prime \prime}$ & $F 814 W$ & $8 \times 1580$ & 24 Nov. 2004 \\
F1 & $00^{\mathrm{h}} 42^{\mathrm{m}} 56^{\mathrm{s}}$ & $+40^{\circ} 50^{\prime} 27^{\prime \prime}$ & $F 814 W$ & $5 \times 1580$ & 25 Nov. 2004 \\
F1 & $00^{\mathrm{h}} 42^{\mathrm{m}} 56^{\mathrm{s}}$ & $+40^{\circ} 50^{\prime} 27^{\prime \prime}$ & $F 814 W$ & $5 \times 1580$ & 10 Dec. 2004 \\
\hline
\end{tabular}

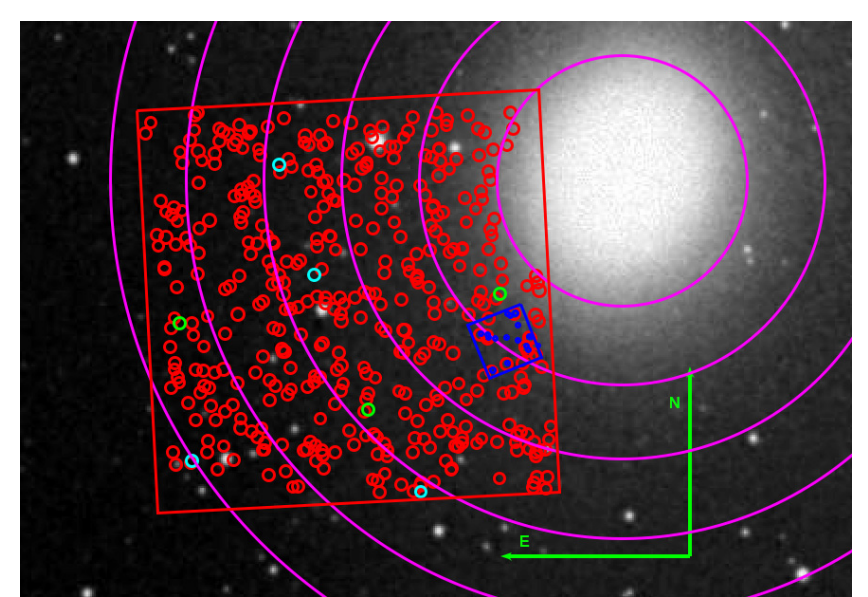

Fig. 1. A $6 \times 5$ arcmin DSS2 where our ACS/WFC field is shown as a large (red) square. The HRC field with its RR Lyr (from Fiorentino et al. 2010b) is shown as a small (blue) square. The RR Lyr, anomalous Cepheid and Population II Cepheid variables detected in the ACS/WFC field are shown as red, cyan and green points, respectively. Circles centred on M 32 used in the density analysis are plotted in magenta.

and extending from 1 to 4.5 arcmin from the centre of M 32, see Fig. 1 and Table 1. The cadence of the images was chosen with the aim of picking up short period variable stars (periods $<1$ day), which includes RR Lyr. The sensitivity of the temporal sampling to these short periods is close to $\sim 100 \%$ complete, for variable stars with periods $<1$ day.

\subsection{Photometric analysis}

We used the same data reduction and analysis techniques described in Fiorentino et al. (2010a,b). The PSF-fitting photometry was carried out using DOLPHOT, a version of HSTphot modified for ACS images (Dolphin 2000b). DOLPHOT returns a time series of measurements for each image as well as a final mean magnitude for all stars found on all the individual frames. DOLPHOT also makes automatic aperture corrections, following the prescription of Sirianni et al. (2005), and corrects for charge transport efficiency (CTE) effects (as described in Dolphin 2000a). Thus we end up with a final catalogue consisting of a time series of 18 individual photometric measurements in two filters, plus mean magnitudes, for $\sim 400000$ stars over the entire ACS/WFC field of view.

There is clearly a very strong gradient in stellar density across our field of view, which increases towards the centre of M 32. This gradient leads to large variations in the crowding of the stellar images. We thus also performed artificial star tests to quantify the completeness of the photometric catalogue over the field of view. A mean completeness above $90 \%$ for the whole field is only found for stars with $m_{F 606 W} \leq 24.5$ mag. For stars fainter than this, the completeness drops rapidly towards the centre of M 32, as the stellar density increases. This has to be carefully corrected before conclusions can be drawn about grandients across this field. We come back to this point in Sect. 5.

We also tested our photometric catalogue for evidence of differential reddening across the field using the well established method of Piotto et al. (1999). We divided our field into 64 $26 \times 26 \operatorname{arcsec}^{2}$ regions and selected one of these as reference. Then for each sub-region we compared the CMD properties with the reference. No evidence for differential reddening was found, down to a limit of $m_{F 606 \mathrm{~W}}=26 \mathrm{mag}$, and thus we adopted a single reddening value of $E(B-V)=0.08 \mathrm{mag}\left(A_{F 606 W}=0.21 \mathrm{mag}\right.$, $A_{F 814 W}=0.13 \mathrm{mag}$, see Fiorentino et al. $2010 \mathrm{~b}$; Bedin et al. 2005), in our analysis.

\subsection{Search for variability}

Details of the procedures to detect and classify variable stars are described in Fiorentino et al. (2010b). The average errors on the periods are $<0.01$ day, on the amplitudes of the variation are $<0.1 \mathrm{mag}$ and on the mean magnitudes are $<0.1 \mathrm{mag}$. We identified 416 bona-fide RR Lyr. We divided the RR Lyr into two groups, two-filters (351 RR Lyr) and one-filter (65 RR Lyr). For two-filters candidates we were able to properly phase the $F 606 \mathrm{~W}$ and $F 814 W$ light curves. For the one-filter candidates we could estimate the period using only one filter (usually $F 606 \mathrm{~W}$ ). We identified also 4 anomalous Cepheids (AC) and 3 Population II Cepheids (P2C), see Sect. 4. In Fig. 2 we show the light curves we have obtained for $2 \mathrm{RR}$ Lyr, $1 \mathrm{AC}$ and $1 \mathrm{P} 2 \mathrm{C}$. We have also detected 61 variable candidates which cannot be accurately classified, they include 27 possible RR Lyr and 34 longer period variables.

We have recovered 9 of the $17 \mathrm{RR} \operatorname{Lyr}(\sim 53 \%)$ found in our previous study with ACS/HRC (Fiorentino et al. 2010b), confirming the low mean completeness $(\sim 57 \%)$ of the HB luminosity on the present ACS/WFC images so close to M 32 .

\section{The RR Lyr variable stars}

From the 416 bona-fide RR Lyr we have detected, we classify 314 as fundamental pulsators ( $F$ or $\mathrm{RR}_{\mathrm{ab}}$ ) and 102 first-overtone pulsators $\left(\mathrm{FO}\right.$ or $\mathrm{RR}_{\mathrm{c}}$ ). Table 2 lists the RR Lyr along with their properties such as position, period, pulsation type classification, mean intensity-weighted magnitudes, amplitudes and dereddened mean magnitudes transformed in the Johnson-Cousin photometric system. The RR Lyrae have been named using their increasing distance from the centre of M 32.

The mean period distribution of the $\mathrm{F}$ pulsators matches a Gaussian peaked at $P_{\mathrm{F}}=0.55$ days with $\sigma=0.07$ days, see Fig. 3, which is consistent with Oosterhoff type I. Similarly, a mean period of $P_{\mathrm{FO}}=0.32$ days with $\sigma=0.04$ days is found for FO pulsators, which is also consistent with an Oosterhoff type I. The ratio between the number of FO and the total sample, $N_{\mathrm{FO}} / N_{\mathrm{TOT}} \sim 0.25$. Our results are thus in agreement with 


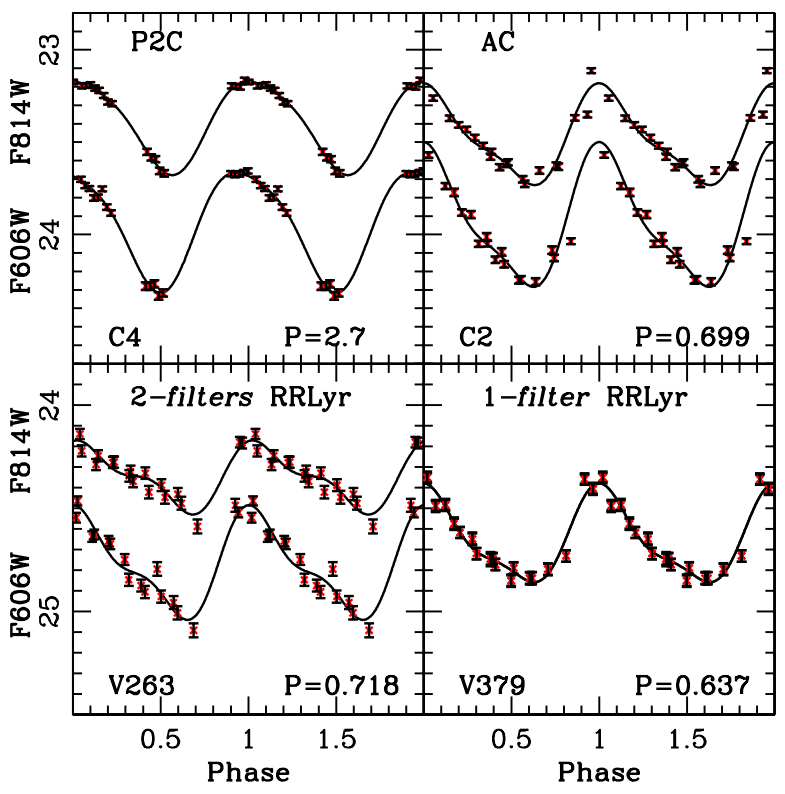

Fig. 2. An example of the quality of the light curves in $F 606 \mathrm{~W}$ and $F 814 W$ filters for four detected variable stars, a Population II Cepheid, an anomalous Cepheid, a one-filter and a two-filters RR Lyr. The ID, type and period have been labeled. The fitting model used to determine the mean magnitude and amplitude has been overplotted. The errorbars represent the photometric error returned by DOLPHOT.

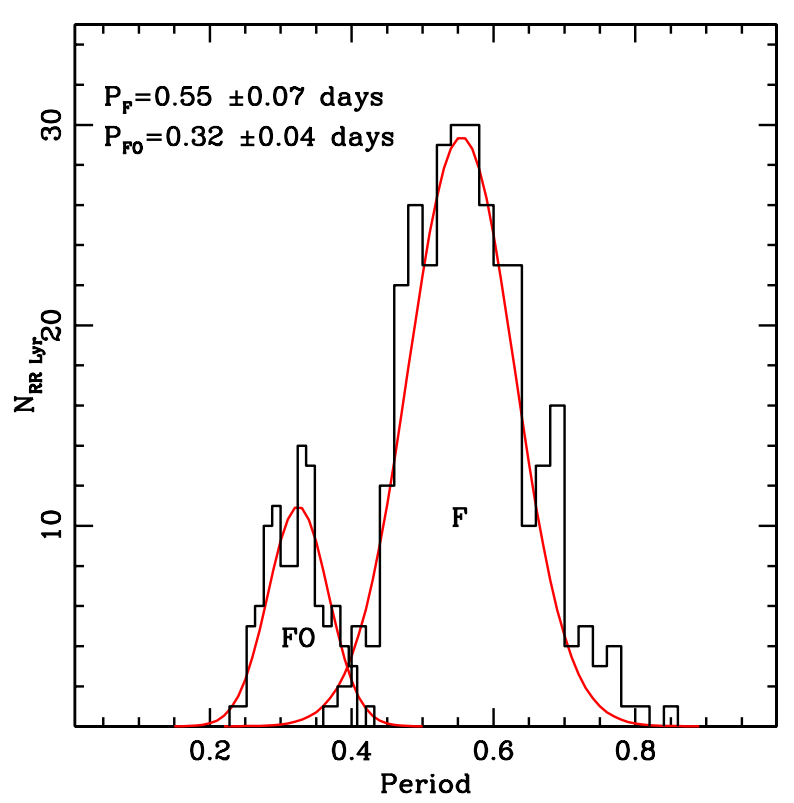

Fig. 3. The period distributions of the fundamental (right) and firstovertone (left) RR Lyr. The periods corresponding to the Gaussian (red) peaks are given as $P_{\mathrm{F}}$ and $P_{\mathrm{FO}}$.

the M 31 study performed by Sarajedini et al. (2009) of two ACS fields close to M 32. Other deep HST/ACS observations of RR Lyr M 31 include six fields of the halo, disk, and giant stellar stream have been studied by Jeffery et al. (2011). The RR Lyr of these fields appear to mostly be of Oosterhoff I type. Comparing our results with these six M 31 fields which sample different components of $\mathrm{M} \mathrm{31}$, the properties of our sample resembles

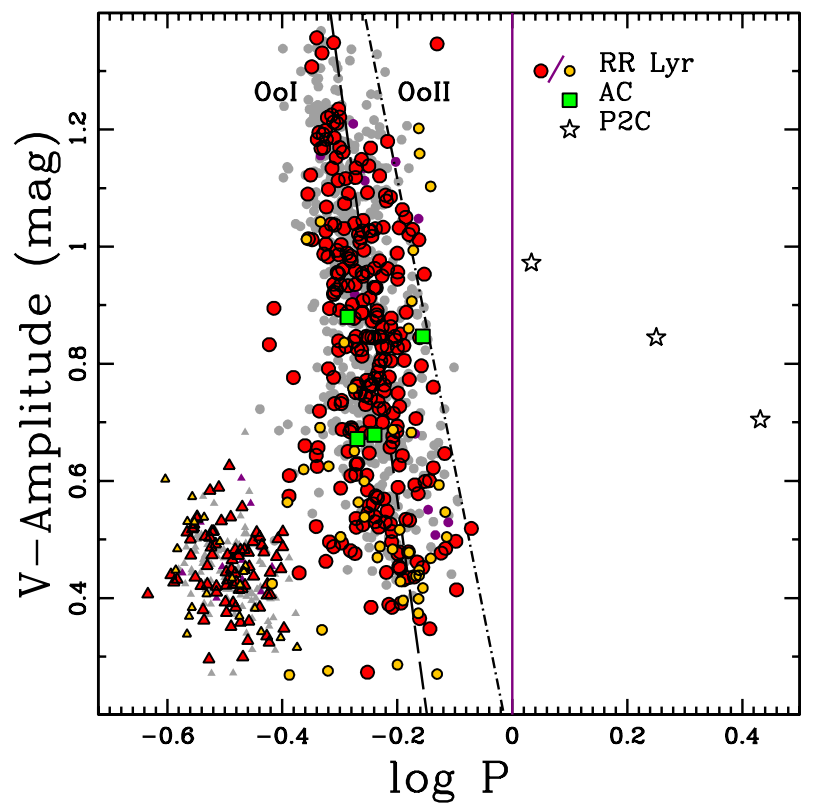

Fig. 4. The $V$ filter amplitude - period $(P)$ Bailey diagram for our observations (coloured symbols) and the observations of M 31 RR Lyr from Sarajedini et al. (2009) and Brown et al. (2004) as grey and magenta symbols. The Oosterhoff type loci, OoI (dashed) and OoII (dot-dashed), are shown (from Clement 2000). For the RR Lyr the triangles are firstovertone pulsators and the circles are fundamental pulsators. RR Lyr two-filters are plotted in red and only in one-filter in orange. Anomalous Cepheids and Population II Cepheids are indicated with squares (green) and stars, respectively.

the giant stream of $\mathrm{M} 31$, where they found $\left\langle P_{\mathrm{F}}\right\rangle=0.56$ and $N_{\mathrm{FO}} / N_{\mathrm{TOT}}=0.33$.

\subsection{Bailey diagram and metallicity}

Figure 4 shows the Bailey diagram of the variable stars detected in the present study overlaid on the M 31 studies of Sarajedini et al. (2009); Brown et al. (2004). This diagram divides into two regions, at $\log P=0$, to distinguish the variable star types. At $\log P<0$ are the RR Lyr and AC and at $\log P>0$ the P2C. The $F 606 \mathrm{~W}$ amplitudes have been transformed to $V$-filter amplitudes (Brown et al. 2004) using $A_{F 606 W}=92 \% A_{V}$ to directly compare the Oosterhoff (Oo) type loci (see Clement 2000, and references therein). In Fig. 4 we see that F RR Lyr are predominantly consistent with an Oo type I, confirming the Oo classification based on the periods alone.

Next, we use this $V$ amplitude-period relation (given by Alcock et al. 2000) to determine the individual metallicities of our entire sample of RR Lyr. This is a method commonly used in the literature for RR Lyr studies without colour information. We found a mean metallicity $[\mathrm{Fe} / \mathrm{H}]=-1.44 \pm 0.55$ dex for our sample, comparable with what was found in M 31. Sarajedini et al. (2009) found $[\mathrm{Fe} / \mathrm{H}]=-1.45 \pm 0.45$ for their $\mathrm{F} 1$ field (the closest to $\mathrm{M} 32$ ) and $[\mathrm{Fe} / \mathrm{H}]=-1.54 \pm 0.43$ for their $\mathrm{F} 2$. In Fig. 5 we show a comparison between individual metallicities for our sample and Sarajedini's sample. We can see that our results are in very good agreement with their studies. We also notice that in our field, and also in the Sarajedini field closest to M 32, there are a few RR Lyr of solar metallicity. This means that we observe more metal-rich RR Lyr than have been found in the halo, disk and giant stream of M 31 by Brown et al. (2004) and 
Table 2. The RR Lyr variable stars we have identified.

\begin{tabular}{ccccccccccc}
\hline \hline ID & $\alpha_{\text {J2000.0 }}$ & $\delta_{\text {J2000.0 }}$ & Period & Type & $\left\langle m_{F 606 W}\right\rangle$ & $A_{F 606 W}$ & $\left\langle m_{F 814 W}\right\rangle$ & $A_{F 814 W}$ & $\left\langle m_{V_{0}}\right\rangle$ & $\left\langle m_{I_{0}}\right\rangle$ \\
\hline V1 & $0: 42: 46.9697$ & $40: 52: 19.159$ & 0.479 & RRab & 24.779 & 0.579 & 23.762 & - & - & - \\
V2 & $0: 42: 47.2873$ & $40: 52: 10.736$ & 0.688 & RRab & 24.707 & 1.113 & 23.712 & - & - & - \\
V3 & $0: 42: 47.0945$ & $40: 52: 27.376$ & 0.690 & RRab & 25.182 & 1.073 & 24.151 & - & - & - \\
V4 & $0: 42: 47.9337$ & $40: 52: 08.733$ & 0.464 & RRab & 24.878 & 0.965 & 25.154 & - & - & - \\
V5 & $0: 42: 48.0864$ & $40: 51: 53.262$ & 0.673 & RRab & 24.674 & 0.920 & 23.701 & - & - & - \\
V6 & $0: 42: 46.1114$ & $40: 51: 02.073$ & 0.722 & RRab & 24.282 & 0.433 & 23.234 & - & - & - \\
V7 & $0: 42: 48.1721$ & $40: 51: 57.548$ & 0.510 & RRab & 24.836 & 0.774 & 23.990 & - & - & - \\
\hline
\end{tabular}

Notes. The mean $F 606 \mathrm{~W}$ and $F 814 \mathrm{~W}$ magnitudes are intensity-weighted over the light curve. $V_{0}$ and $I_{0}$ are the de-reddened mean magnitudes in the Johnson-Cousin photometric system obtained following the prescriptions by Sirianni et al. (2005). Type classification, $R_{a b}$ and $R_{c}$, indicate fundamental and first-overtone pulsation modes, respectively. ${ }^{(a)}$ For one-filter RR Lyr we have only one mean magnitude, thus we can not estimate the corresponding amplitude or Johnson-Cousin magnitudes, we can only indicate the Dolphot mean magnitude. The full table is available at the CDS.

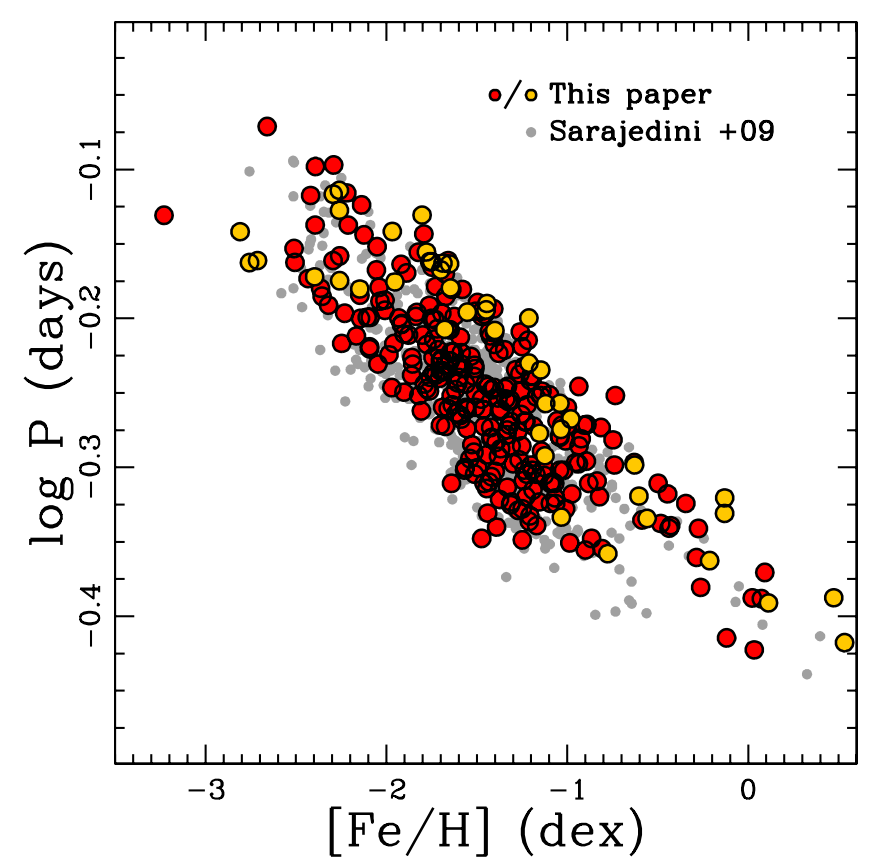

Fig. 5. The metallicity $([\mathrm{Fe} / \mathrm{H}])$ - period $(P)$ distribution for our sampled fundamental RR Lyr. The colour code is the same used in Fig. 4. Undelying our observation those os Sarajedini et al. (2009) are shown as grey symbols.

Jeffery et al. (2011), where using the same method, no RR Lyr with metallicity larger than $[\mathrm{Fe} / \mathrm{H}]>-1$ dex have been found.

\subsection{The horizontal branch}

The horizontal branch (HB) is almost invisible in our CMD, because it is sparsely populated and dominated by contamination from other populations, such as an extensive and broad RGB and a young $\mathrm{BP}$ of stars in the main sequence region. The only way to reliably identify the HB is using RR Lyr. In Fig. 6a we show the CMD of the horizontal branch region for our two-filters RR Lyr, for which we have light-curve weighted mean magnitudes and colours. The RR Lyr define the HB and thus give us the opportunity to determine the mean distance modulus of the sample. To derive the distance modulus, we transformed mean $m_{F 606 W}$ and $m_{F 814 W}$ (Cols. 8 and 10 in Table 2) into Johnson-Cousins $m_{V}$ and $m_{I}$ (Cols. 12 and 13 in Table 2). We thus obtained a de-reddened $V$ magnitude of the $\mathrm{HB},\left\langle V_{0}\right\rangle=24.95 \pm 0.18 \mathrm{mag}$. Then, assuming the average metallicity found above and using the $M_{V}-[\mathrm{Fe} / \mathrm{H}]$ relationship (Carretta et al. 2000), we obtain a distance modulus of $\mu_{0}=24.33 \pm 0.21 \mathrm{mag}$. The large uncertainty is due to both the large metallicity spread (see Fig. 5) and the large scatter on the HB $( \pm 0.5 \mathrm{mag})$, as seen in Fig. 6 a.

In Fig. 6b, we show the luminosity histogram of our twofilters RR Lyr and we compare it with that for field F1 from Sarajedini et al. (2009). The peak of our luminosity distribution is brighter than Sarajedini's by $\sim 0.16$ mag. This could be due to the significantly different surface brightness of the two fields, which is $\sim 22 \mathrm{mag} / \operatorname{arcsec}^{2}$ in our field and $\sim 25 \mathrm{mag} / \mathrm{arcsec}^{2}$ in Sarajedini's. A brighter surface brightness implies more severe blending effects that can lead to brighter magnitudes for individual stars (e.g., Fiorentino et al. 2010b). However, the broad spread we find in $m_{F 606 W}$ does not change using only the least blended $^{2}$ RR Lyr in our sample. This suggests that this spread is not likely to be caused by photometric errors. The spread could be due to either the RR Lyr being at a range of different distances and/or having a large range in metallicity. Note that in both cases differences in the evolutionary states may affect the RR Lyr luminosity, which would further increase the final HB luminosity range. In Fig. 6a we show the effect of a metallicity range using the theoretical HB tracks for a range of metallicity at the mean distance modulus derived above. The metallicity spread inferred for the RR Lyr is consistent with the large range derived from the periods and amplitudes in Fig. 4. However this does not account for the whole HB spread. In addition, a distance spread is also very likely because the M 32 field is mixed with M 31 population. Assuming the mean metallicity found above and moving the relative zero age HB locus to fit the brightest and the faintest RR Lyr in Fig. 6a, we find a distance modulus of $24.33_{-0.43}^{+0.47}$ mag (or $d=734_{-131}^{+178} \mathrm{Kpc}$ ).

To determine how many, if any, of the RR Lyr in our sample are likely to be associated with M 32, we need to look at their radial distribution from the centre of M 32.

\section{The Cepheid variable stars}

In our sample we have identified 7 variable stars (described in Table 3) with luminosities significantly higher than the HB, as shown in Fig. 6. These stars are at luminosities and colours consistent with both AC and P2C (Fiorentino et al. 2006; Di Criscienzo et al. 2007). The AC are core-He burning stars of intermediate age ( 1-6 Gyr old), with mass of 1-2 $M_{\odot}$, and they cover a period range from a few hours to $\sim 2$ days. The P2C are lower mass stars $\left(\leq 0.8 M_{\odot}\right)$ that just finished their core-He burning phase and come from the blue HB. They are very old

2 As defined by the DOLPHOT crowding parameter. 

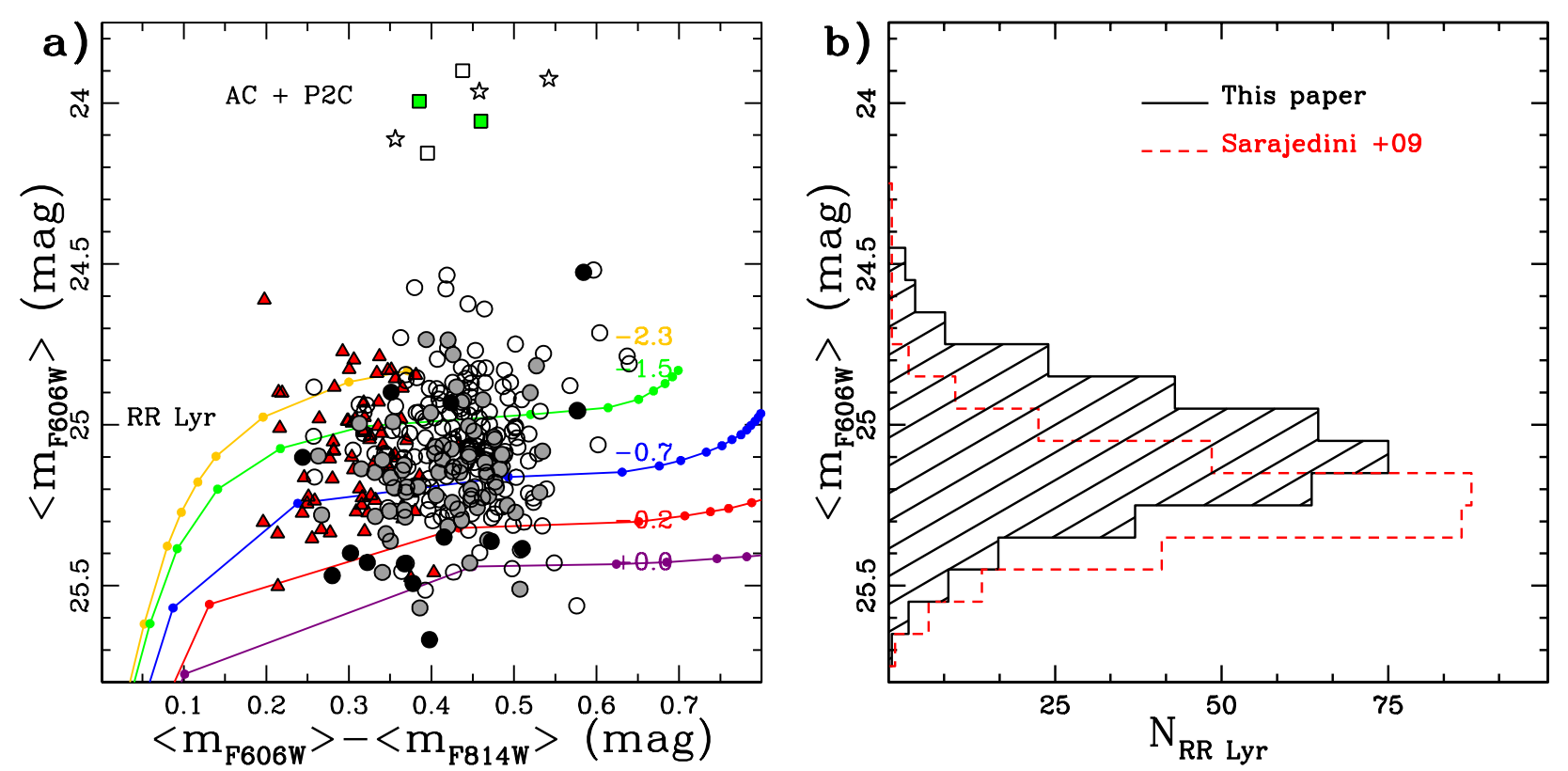

Fig. 6. a) The RR Lyr region of the CMD, where only variable stars detected in two filters, with well defined colours and magnitudes, are shown. Red triangles are the same as in Fig. 2a, but the colouring of the F pulsators is determined by their metallicities, where empty circles are metal-poor $([\mathrm{Fe} / \mathrm{H}] \leq-1.5 \mathrm{dex})$, grey circles are metal-intermediate $(-0.5 \geq[\mathrm{Fe} / \mathrm{H}] \geq-1.5 \mathrm{dex})$ and filled circles are metal-rich $([\mathrm{Fe} / \mathrm{H}] \geq-1 \mathrm{dex})$. AC and P2C are indicated with squares and stars, respectively. AC pulsating in first-overtone mode are highlighted with filled colour (green). The coloured lines are HB tracks from (Pietrinferni et al. 2004) for different metallicities, as labeled. b) The luminosity function for our 2-filter RR Lyr sample (black solid line) compared with that found by Sarajedini et al. (2009) (red dashed line).

Table 3. The confirmed Cepheid variable stars.

\begin{tabular}{ccccccccccc}
\hline \hline ID & $\alpha_{\text {J2000.0 }}$ & $\delta_{\text {J2000.0 }}$ & Period & Type & $\left\langle m_{F 606 W}\right\rangle$ & $A_{F 606 W}$ & $\left\langle m_{F 814 W}\right\rangle$ & $A_{F 814 W}$ & $\left\langle m_{V_{0}}\right\rangle$ & $\left\langle m_{I_{0}}\right\rangle$ \\
\hline C1 & $0: 42: 47.8102$ & $40: 50: 53.238$ & 1.78 & P2C & 23.964 & 0.782 & 23.506 & 0.579 & 23.839 & 23.368 \\
C2 & $0: 42: 56.4848$ & $40: 51: 06.308$ & 0.70 & AC-F & 23.899 & 0.784 & 23.462 & 0.551 & 23.765 & 23.323 \\
C3 & $0: 42: 57.9929$ & $40: 52: 04.630$ & 0.53 & AC-FO & 23.995 & 0.622 & 23.610 & 0.421 & 23.853 & 23.471 \\
C4 & $0: 42: 54.1987$ & $40: 49: 53.248$ & 2.70 & P2C & 23.925 & 0.652 & 23.382 & 0.506 & 23.835 & 23.244 \\
C5 & $0: 43: 02.8871$ & $40: 50: 43.142$ & 1.08 & P2C & 24.112 & 0.900 & 23.756 & 0.627 & 23.962 & 23.618 \\
C6 & $0: 42: 51.8780$ & $40: 49: 07.727$ & 0.57 & AC-FO & 24.057 & 0.629 & 23.597 & 0.412 & 23.909 & 23.448 \\
C7 & $0: 43: 02.5661$ & $40: 49: 28.127$ & 0.51 & AC-F & 24.156 & 0.815 & 23.761 & 0.547 & 24.017 & 23.622 \\
\hline
\end{tabular}

Notes. Same as in Table 2.

( $>10$ Gyr) and typically have periods $>1$ day. Those with periods $<3$ days, are the so-called BL Hercules variable stars and they come from the warm part of the HB (e.g. Marconi et al. 2011).

The overlap in the period ranges of $\mathrm{AC}$ and $\mathrm{P} 2 \mathrm{C}$ makes a proper distinction between them complicated. We have plotted them all in the Bailey diagram, see Fig. 4, where we see that three of them occupy the region with periods $>1$ day, and the remaining four lie on the RR Lyr period-amplitude relation. In Fig. 7 (lower panel) we plot the pulsation Instability Strip derived from theoretical models (Marconi et al. 2004) for AC, assuming the distance modulus derived in the previous section $\left(\mu_{0}=24.33\right)$. There is only one variable not within the instability strip, and this corresponds to the longest period, namely $\mathrm{C} 4$. To check that the Cepheids within the instability strip are genuine $\mathrm{AC}$, we also use the colour information plotting them in the reddening-free Wesenheit plane, i.e. WES $(V$, $V-I)=V-2.54 E(V-I)$ see top panel of Fig. 7. In this plane the separation between these two classes of variables is very clear. The P2C follow a well established period-luminosity relation, whereas the AC follow a more spread out period-luminosity relation that depends on stellar mass. In Fig. 7 (top panel) we also show the theoretical Wesenheit relations (Fiorentino et al. 2007; Di Criscienzo et al. 2007) for both AC and P2C. Thus we can classify three Cepheids $(\mathrm{C} 4, \mathrm{C} 6, \mathrm{C} 7)$ as P2C and the other four as AC (see Table 3).

We can constrain the masses for the four AC, using our theoretical approach (extensively described in Marconi et al. 2004; Caputo et al. 2005; Fiorentino et al. 2006) which allow us to simultaneusly derive the mass and the pulsation mode of an AC using the pulsation properties. We use the well defined mass dependent period-magnitude-amplitude relation, which is valid only for $\mathrm{F}$ pulsator, and the period-magnitude-colour relations which are available for both $\mathrm{F}$ and $\mathrm{FO}$ pulsators. Applying these relations to our four AC we classify two of them F pulsators and the remaining FO pulsators (see Table 3 ) with masses in the range $1.2-1.9 M_{\odot}$. Their masses indicate these stars come from a stellar population with ages between 1 and 4 Gyr old.

We notice that the distance modulus found from RR Lyr agrees well with that from the P2C Wesenheit relation. The only 


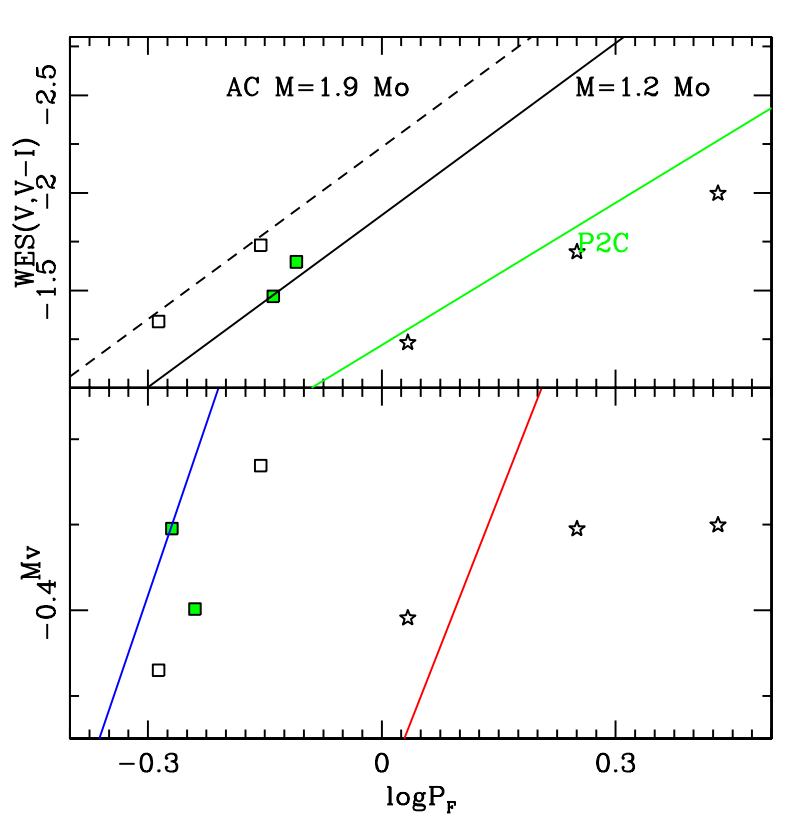

Fig. 7. Bottom: anomalous Cepheids (squares) and Population II Cepheids (stars) in the $M_{V}$-period $(P)$ plane. First-overtone AC have been highlighted with filled colour (green). It is not possible to make a clear classification between these two class of variables. We have plotted the cold (red) and the warm (blue) edges of the pulsation instability strip as derived by theoretical models (Fiorentino et al. 2006). Top: wesenheit plane, where the separation between the anomalous Cepheids and Population II Cepheids is clearer. The green solid line represents the relation for Population II Cepheids, and the black solid and dashed line represent the mass dependent Wesenheit relations for anomalous Cepheids, with masses $1.2 M_{\odot}$ and $1.9 M_{\odot}$ respectively. The distance modulus assumed is 24.33, as we found from RR Lyr.

point of concern is the low luminosity of the longest period $\mathrm{P} 2 \mathrm{C}$, $\mathrm{C} 4$. However mean luminosities and amplitudes could be affected by the small temporal coverage used to sample the full pulsation cycle for these relatively long period variable stars.

\section{Radial distributions of stellar populations}

The stellar populations of M 31 and M 32 are so similar and mixed, in our field, that the characteristics of M 32 can only be defined by looking for features that follow the strong concentration of stars towards the centre of M 32. This has to be done with extreme care, because the crowding properties of the images are also changing closer to the centre of M 32. We are mainly concerned to determine if there is a RR Lyr population that can be reliably identified with $\mathrm{M} 32$. To this end it is also useful to look at the radial distribution of other stellar populations that can be identified in our CMD.

In Fig. 8 we show the CMD for all the detected stars in our ACS/WFC field of view. The two over-plotted isochrones are $8 \mathrm{Gyr}$ old, which is the mean age for the bulk of the stellar population (Monachesi et al. 2011). To look for spatial gradients over our field towards the centre of M 32, we select different stellar populations from the CMD, as follows:

- i) the bright BP, which are young $\leq 500$ Myr old main sequence stars;

- ii) an intermediate BP, which includes a contribution from young main sequence stars $(\leq 1 \mathrm{Gyr}$ old) and also the blue $\mathrm{HB}$;

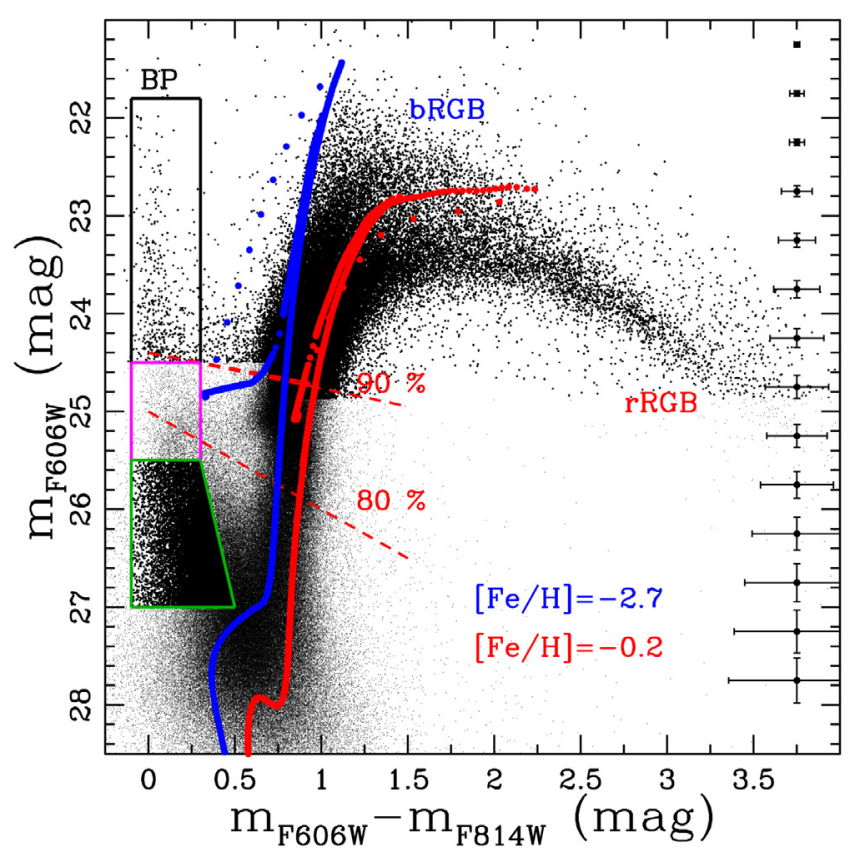

Fig. 8. The ACS/WFC CMD, for all the detected stars. The mean completeness levels (red dashed lines) and the photometric errors are shown. Over-plotted are 8 Gyr old reddened isochrones, from Pietrinferni et al. (2004) with $[\mathrm{Fe} / \mathrm{H}]=-2.7 \mathrm{dex}$ (blue) and $-0.2 \mathrm{dex}$ (red). Distance modulus and the reddening used are $\mu_{0}=24.33$ and $E(B-V)=$ $0.08 \mathrm{mag}$. Isochrones and boxes have been used to select the populations. The blue (bRGB) and red RGB (rRGB) on either side of $[\mathrm{Fe} / \mathrm{H}]=-0.2$ dex isochrone and the blue plume (BP) which is separated into bright (black box), intemediate (magenta box) and faint (dark green box) components.

- $\quad$ iii) the faint BP, which is made up of main sequence stars $\leq 4$ Gyr old;

- iv) RR Lyr;

- v) the blue RGB, which is those stars with $m_{F 606 W} \leq$ 24.9 mag that lie on the RGB between the blue and the red isochrones and they should be mostly old metal-poor stars $(-2.7 \leq[\mathrm{Fe} / \mathrm{H}] \leq-0.2)$, but could also include younger $(<8$ Gyr old) metal-rich stars $([\mathrm{Fe} / \mathrm{H}] \leq-0.2)$;

- vi) the red RGB, which is those stars with $m_{F 606 W} \leq$ 24.9 mag and on the red side of the red RGB and are all metal-rich RGB stars.

These populations have been chosen to trace different ages and metallicities in the CMD attempting to separate different contributions coming from M 31 and/or M 32. In fact, we do not expect that young stars with ages less than $0.5 \mathrm{Gyr}$ belong to an elliptical galaxy such as M 32. On the other hand, we expect that the contribution to the metal-rich component, as the red RGB, is mostly coming from M 32, being almost invisible in other accurate HST CMDs of the disk/halo components of M 31 (e.g. Brown et al. 2006, and reference therein).

We expect that the completeness will vary along with the distance from the centre of M 32. For each selected population we have computed the completeness in annuli around M32, as defined in Fig. 1. To account for the large colour baseline of our CMD, we computed the completeness for all the blue stars ( $V-I$ less than $\sim 0.7 \mathrm{mag}$ ) and for all the red stars $(V-I$ larger than $\sim 0.7 \mathrm{mag}$ ) independently. As an example, at the luminosity of the RR Lyr on the HB $\left(m_{F 606 W} \sim 25.07 \mathrm{mag}\right)$ the mean completeness for RR Lyr is $57 \%$ in the inner annulus 


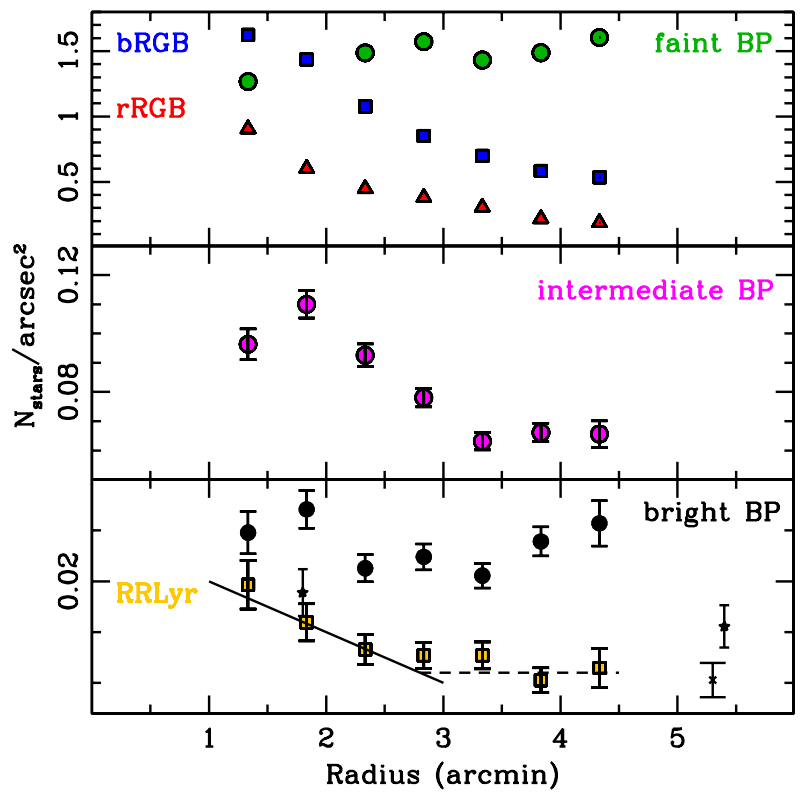

Fig. 9. The variation of the fraction of stars with the distance from the centre of M 32 for the populations selected from the CMD. The colour code is the same used in Fig. 8. The poissonian errorbars are plotted where they are larger than the symbols. Top panel, the bRGB, the rRGB and the faint BP have been plotted with circles, squares and stars, respectively. In the middle panel the intermediate BP distribution is plotted. The bottom panel shows the RR Lyr and the bright BP radial distributions with squares and circles, respectively. The bright BP circles have been shifted of +0.01 , to make the plot more clear. A comparison with previous RR Lyr detections is also shown with stars (F1 and F2 Fiorentino et al. 2010b) and cross (F1 from Sarajedini et al. 2009). Solid and dashed lines represent the fit to M 32 and M 31 profiles, respectively.

( $r \sim 1.3$ arcmin), whereas it increases to $85 \%$ in the outer annulus $(r \sim 4.3$ arcmin).

In Fig. 9, we show the number of stars per $\operatorname{arcsec}^{2}$ of the selected stellar populations corrected for the completeness, as determined by artificial star tests. Four out of the six selected populations, i.e. the blue and red RGB, the intermediate BP and the RR Lyr, increases going to the centre of M 32 defining the radial profile of the elliptical galaxy. On the contrary, the faint and bright BPs (top panel) show, if anything, a shallow opposite trend, suggesting an association to M 31. Stars so young are a clear feature of the M 31 field from other ACS/WFC studies, and this suggests that all the young stars observed in our field are likely associated to M 31. However, the number statistics are low and it has been suggested by Monachesi et al. (2011) that there is a BP component in M 32 (with ages $\geq 1$ Gyr). We do not confirm this finding, however it could easily be hidden in the huge contamination from M 31. We note that, among the $\mathrm{BP}$ components only the intermediate $\mathrm{BP}$, which includes also stars from the HB, increases going towards the centre of M 32 (middle panel in Fig. 9) then supporting the presence of the HB in M 32. If there were young stars in M 32 then also the other BP components should show the same signature, but they do not.

Finally, the RR Lyr distribution found in the present study shows an increasing trend towards the centre of M 32 and confirms previous results (Alonso-García et al. 2004; Fiorentino et al. 2010b). In Fig. 9 we also showed the RR Lyr detections found from Sarajedini et al. (2009) and Fiorentino et al. (2010b), which are in very good agreement with the RR Lyr radial profile found in this paper. Our previous RR Lyr detections, two points from Fiorentino et al. (2010b), were uncertain because of their large poissonian errors due to the very small FoV $(30 \times 30$ arcsec) of ACS/HRC. Our new detection of 416 RR Lyr and their spatial variation over the $3.3^{\prime} \times 3.3^{\prime} \mathrm{ACS} / \mathrm{WFC}$ field of view is the strongest evidence obtained so far for an ancient population in M 32.

We also notice that the RR Lyr distribution is quite flat going from outside to the centre of M 32 up to about 3 arcmin, suggesting that what we are observing for radii larger than 3 arcmin is a high background from M 31. Something similar can be seen also in the intermediate BP distribution supporting the idea of the strong M 31 background.

We can attempt an estimation of the fraction of the RR Lyr that belong to M 32 by assuming that the RR Lyr outside 3 arcmin from the centre of M 32 represent the M 31 background $\left(N_{\text {stars }} / \operatorname{arcsec}^{2}=0.011\right.$, dashed line in Fig. 9). Then, we can fit a slope to the remaining observations ( $r \leq 3 \mathrm{arcmin})$, $N_{\text {stars }} / \operatorname{arcsec}^{2}=0.025-0.005^{*} r$ (solid line in Fig. 9). The total number of stars whithin 3 arcmin from the centre to $M 32$ is 327 RR Lyr, which includes the background from M 31. Then we can estimate the number of RR Lyr inside the 3 arcmin, subtracting the contribution expected from the background of M 31 . At least $\sim 83$ RR Lyr could to be associated to M 32 .

\section{Conclusions}

The radial density of RR Lyr in our HST/WFC sample clearly increases towards the centre of M 32. This convincingly shows that we have identified an ancient stellar population of at least 83 RR Lyr associated to M 32. These new RR Lyr detections are in agreement with our previous results (Fiorentino et al. 2010b), which is actually a bit lucky given the statistics of the small field of view of HST/HRC.

The same trend towards the centre of M 32 is also followed by the other stellar components. Such as the blue and the red component of the RGB. This suggests that M 32 contains both a moderately metal-poor $(-2.7 \leq[\mathrm{Fe} / \mathrm{H}] \leq-0.2)$ and a metal-rich $([\mathrm{Fe} / \mathrm{H}] \geq-0.2)$ stellar population.

On the other hand, the bright and the faint blue plume of young main sequence stars ( $\leq 4$ Gyr old) has a more smooth distribution suggesting that it is associated exclusively with the M 31 background. This result suggests that the $4 \mathrm{AC}$, with masses from 1.2 to $1.9 M_{\odot}$, found in our analysis are likely to be associated to M 31.

We find compelling evidence for a large spread in the metallicities of the RR Lyr in our sample. They span $-2.4 \leq[\mathrm{Fe} / \mathrm{H}] \leq$ 0 dex with a mean metallicity of $\langle[\mathrm{Fe} / \mathrm{H}]\rangle \sim-1.44 \pm 0.55$ dex.

The RR Lyr in this study are classified as Oosterhoff type I, in agreement with recent results (Brown et al. 2004; Jeffery et al. 2011) for RR Lyr observed in M 31. The properties of our RR Lyr sample resemble those of the stream of M 31. This could suggest that M 32 could have contributed to the M 31 stream with its old stellar population, or that the stream dominates M 31 at the position of M 32 .

Acknowledgements. We thank A. Dolphin and D. Weisz for their technical support running DOLPHOT on many WFC/ACS images simultaneously. We are grateful to A. Mucciarelli for his help in calculating the reddening map of our WFC/ACS field. We thank S. Cassisi, M. Di Criscienzo, G. Greco, M. Bellazzini and G. Bono for useful discussions. G.F. particularly thanks R. Merighi his technical support. The authors acknowledge an anonymous referee for his/her suggestions that improved the content and the readability of the manuscript. G.F. and E.T. have been supported by a NWO-VICI grant and GF by the INAF fellowship 2009 grant. E.T. thanks the hospitality of Observatiore de la Côte d'Azur. 
A\&A 539, A138 (2012)

\section{References}

Alcock, C., Allsman, R. A., Alves, D. R., et al. 2000, AJ, 119, 2194 Alonso-García, J., Mateo, M., \& Worthey, G. 2004, AJ, 127, 868 Bedin, L. R., Cassisi, S., Castelli, F., et al. 2005, MNRAS, 357, 1038 Brown, T. M., Ferguson, H. C., Smith, E., et al. 2004, AJ, 127, 2738 Brown, T. M., Smith, E., Ferguson, H. C., et al. 2006, ApJ, 652, 323 Caputo, F., Bono, G., Fiorentino, G., Marconi, M., \& Musella, I. 2005, ApJ, 629 1021

Carretta, E., Gratton, R. G., Clementini, G., \& Fusi Pecci, F. 2000, ApJ, 533, 215

Clement, C. M. 2000, in The Impact of Large-Scale Surveys on Pulsating Star

Research, ed. L. Szabados, \& D. Kurtz, ASP Conf. Ser., 203, IAU Colloq. 176,266

Clementini, G., Contreras, R., Federici, L., et al. 2009, ApJ, 704, L103

Coelho, P., Mendes de Oliveira, C., \& Fernandes, R. C. 2009, MNRAS, 396, 624

Di Criscienzo, M., Caputo, F., Marconi, M., \& Cassisi, S. 2007, A\&A, 471, 893

Dolphin, A. E. 2000a, PASP, 112, 1397

Dolphin, A. E. 2000b, PASP, 112, 1383

Dolphin, A. E., Saha, A., Olszewski, E. W., et al. 2004, AJ, 127, 875
Fiorentino, G., Limongi, M., Caputo, F., \& Marconi, M. 2006, A\&A, 460, 155 Fiorentino, G., Marconi, M., Musella, I., \& Caputo, F. 2007, A\&A, 476, 863 Fiorentino, G., Contreras Ramos, R., Clementini, G., et al. 2010a, ApJ, 711, 808 Fiorentino, G., Monachesi, A., Trager, S. C., et al. 2010b, ApJ, 708, 817 Grillmair, C. J., Lauer, T. R., Worthey, G., et al. 1996, AJ, 112, 1975 Jeffery, E. J., Smith, E., Brown, T. M., et al. 2011, AJ, 141, 171 Marconi, M., Fiorentino, G., \& Caputo, F. 2004, A\&A, 417, 1101 Marconi, M., Bono, G., Caputo, F., et al. 2011, ApJ, 738, 111

Monachesi, A., Trager, S. C., Lauer, T. R., et al. 2011, ApJ, 727, 55

Pietrinferni, A., Cassisi, S., Salaris, M., \& Castelli, F. 2004, ApJ, 612, 168 Piotto, G., Zoccali, M., King, I. R., et al. 1999, AJ, 118, 1727

Pritchet, C. J., \& van den Bergh, S. 1987, ApJ, 316, 517

Rejkuba, M., Harris, W. E., Greggio, L., \& Harris, G. L. H. 2011, A\&A, 526, A123

Renzini, A. 2006, ARA\&A, 44, 141

Rose, J. A. 1994, AJ, 107, 206

Sarajedini, A., Mancone, C. L., Lauer, T. R., et al. 2009, AJ, 138, 184

Sirianni, M., Jee, M. J., Benítez, N., et al. 2005, PASP, 117, 1049

Trager, S. C., Faber, S. M., Worthey, G., \& González, J. J. 2000, AJ, 119, 1645 